\section{O Programa de Saúde da Família e a construção de um novo modelo para a atenção básica no Brasil}

\author{
Sarah Escorel, ${ }^{1}$ Ligia \\ Giovanella, ${ }^{1}$ Maria Helena \\ Magalhães de Mendonça ${ }^{1} e$ \\ Mônica de Castro Maia Senna ${ }^{2}$
}

A implantação do Sistema Único de Saúde (SUS) no Brasil a partir da década de 1990 representou uma importante inflexão no padrão historicamente consolidado de organização dos serviços de saúde no país. Financiado com recursos fiscais, o SUS fundamentou-se em três princípios básicos: i) universalidade do acesso aos serviços em todos os níveis de assistência para todos os cidadãos brasileiros, independentemente de renda, classe social, etnia, ocupação e contribuição; ii) descentralização em direção aos estados e municípios, com redefinição das atribuições e responsabilidades dos três níveis de governo; e iii) participação popular na definição da política de saúde em cada nível de governo, bem como no acompanhamento de sua execução. Na segunda metade da década, o processo de implantação do SUS caminhou pari passu à adoção de uma série de medidas governamentais voltadas para o fortalecimento da atenção básica de saúde, entendida pelo Ministério da Saúde como "um conjunto de ações, de caráter individual ou coletivo, situadas no primeiro nível de atenção dos sistemas de saúde, voltadas para a promoção da saúde, a prevenção de agravos, o tratamento e a reabilitação" (1). Após décadas de privilégio à atenção hospitalar, herança da medicina previdenciária, em que a alocação de recursos federais em estados e municípios se dava com base principalmente na produção de serviços e na capacidade instalada, os esforços, programas e investimentos públicos passaram a se concentrar na atenção básica, com a adoção do Programa de Saúde da Família (PSF), por meio de incentivos financeiros específicos e da criação de mecanismos de transferência de recursos federais calculados com base no número de habitantes de cada município (per capita).

A implantação do PSF é um marco na incorporação da estratégia de atenção primária na política de saúde brasileira. A doutrina de cuidados primários de saúde da conferência de Alma-Ata já havia, anteriormente, influenciado a formulação das políticas de saúde no Brasil (2), e seus princípios foram traduzidos no novo modelo de proteção social em saúde instituído com o SUS. Contudo, uma política específica, nacional, de atenção primária para todo o país nunca havia sido formulada, ainda que diversas experiências localizadas tenham sido implementadas de modo disperso.

Surgido em 1994 e inicialmente voltado para estender a cobertura assistencial em áreas de maior risco social, o PSF aos poucos adquiriu centralidade 
na agenda governamental. Desde 1999, passou a ser considerado pelo Ministério da Saúde como uma estratégia estruturante dos sistemas municipais de saúde, com vistas a reorientar o modelo assistencial e imprimir uma nova dinâmica na organização dos serviços e ações de saúde (3). A estratégia de saúde da família incorpora os princípios do SUS e se aproxima dos pressupostos da atenção primária em saúde dimensionados por Starfield (4) (primeiro contato, longitudinalidade, abrangência do cuidado, coordenação e orientação à família e às comunidades), buscando romper com a noção de uma atenção de baixo custo simplificada.

O modelo preconiza uma equipe de saúde da família de caráter multiprofissional (médico generalista, enfermeiro, auxiliar de enfermagem e agente comunitário de saúde) que trabalha com definição de território de abrangência, adscrição de clientela, cadastramento e acompanhamento da população residente na área. Pretende-se que a unidade de saúde da família constitua a porta de entrada ao sistema local e o primeiro nível de atenção, o que supõe a integração à rede de serviços mais complexos. Recomenda-se que cada equipe fique responsável por entre 600 e 1000 famílias (2 400 a 4500 habitantes). A equipe deve conhecer as famílias do seu território de abrangência, identificar os problemas de saúde e as situações de risco existentes na comunidade, elaborar um programa de atividades para enfrentar os determinantes do processo saúde/doença, desenvolver ações educativas e intersetoriais relacionadas com os problemas de saúde identificados e prestar assistência integral às famílias sob sua responsabilidade no âmbito da atenção básica.

Ao longo da década, o PSF expandiu-se em todo o país sob indução do Ministério da Saúde, atingindo, em junho de 2004, 84\% dos municípios brasileiros, com 20561 equipes e cobertura populacional de $38 \%$ (cerca de 60 milhões de habitantes) (5). Um olhar sobre as experiências em curso demonstra uma grande diversidade vis-à-vis as imensas disparidades inter e intra-regionais e as enormes desigualdades sociais que marcam a realidade brasileira $(6,7)$. A implantação do PSF foi acelerada nos municípios de pequeno porte, com baixa ou nenhuma capacidade instalada. Todavia, nos grandes centros urbanos, a implementação do PSF tem sido lenta, pois deparou-se com questões de maior complexidade relacionadas à concentração demográfica, ao elevado grau de exclusão no acesso aos serviços de saúde, a agravos de saúde característicos das grandes cidades e metrópoles e a uma oferta consolidada em uma rede assistencial desarticulada e mal distribuída (8).

A estratégia de saúde da família encerra, em sua concepção, mudanças na dimensão organizacio- nal do modelo assistencial ao constituir uma equipe multiprofissional responsável pela atenção à saúde de uma população circunscrita, definir o generalista como o profissional médico de atenção básica e instituir novos profissionais, a saber, os agentes comunitários de saúde, voltados para a ação comunitária, ampliando a atuação da equipe sobre os determinantes mais gerais do processo saúde-enfermidade. Todavia, por ser uma iniciativa recente, poucos são os estudos que permitem conhecer se de fato ocorreram mudanças substantivas no modelo assistencial a partir de sua introdução. Passaremos, então, a analisar as possíveis mudanças no modelo de atenção à saúde introduzidas pela implantação do PSF, tendo como foco a constituição do PSF como porta de entrada no sistema de saúde, sua articulação com uma rede de serviços de saúde integrada e a incorporação de novas práticas assistenciais. A análise toma por base os resultados de uma pesquisa desenvolvida pelo Núcleo de Estudos Político-Sociais em Saúde da Escola Nacional de Saúde Pública, Fundação Oswaldo Cruz (NUPES/ DAPS/ENSP/FIOCRUZ), que teve por objetivo examinar fatores facilitadores e limitantes da implementação do PSF em grandes centros urbanos (9). ${ }^{3}$

\section{ESTUDOS DE CASO}

Foram estudados os casos de 10 municípios com população acima de 100000 habitantes de quatro regiões do país. A seleção foi feita de forma a incluir situações diversificadas de implantação do PSF e detectar o maior número de fatores limitantes e facilitadores do processo. Foram selecionadas experiências bem-sucedidas, municípios que apresentavam dificuldades ou singularidades e cidades nas quais o programa encontrava-se em fase inicial de implantação, com coberturas entre 11 e $81 \%$ da população municipal. A tabela 1 mostra as características das cidades onde os estudos de caso foram realizados $(9,10)$.

A pesquisa de que fazem parte os resultados do presente estudo contemplou as perspectivas e experiências dos gestores, dos profissionais das equipes de saúde da família e das famílias cadastradas, o que denotou um extenso trabalho de campo nos municípios selecionados. Foram realizadas 110 entrevistas semi-estruturadas com gerentes das secretarias municipais e estaduais de saúde e do PSF e com conselheiros municipais de saúde, representantes dos usuários. Foi realizado um inquérito com

\footnotetext{
3 A pesquisa recebeu apoio financeiro do Ministério da Saúde e teve por objetivo oferecer subsídios ao desenvolvimento do Projeto de Expansão da Saúde da Família em grandes centros urbanos (PROESF) do Departamento de Atenção Básica da Secretaria de Políticas de Saúde do Ministério da Saúde (DAB/SPS/MS).
} 
TABELA 1. Características demográficas e da implantação do Programa de Saúde da Família nas cidades onde foram realizados estudos de caso, Brasil, 2000

\begin{tabular}{|c|c|c|c|c|c|}
\hline Município a & População & $\begin{array}{c}\text { Densidade } \\
\left(\mathrm{hab} / \mathrm{km}^{2}\right)\end{array}$ & $\begin{array}{c}\text { Ano de } \\
\text { implantação } \\
\text { do programa }\end{array}$ & $\begin{array}{l}\text { No. de } \\
\text { equipes }^{b}\end{array}$ & $\begin{array}{c}\text { Cobertura } \\
(\%)^{c}\end{array}$ \\
\hline Camaragibe (PE) & 128702 & 2681,29 & 1994 & 31 & 81 \\
\hline Palmas (TO) & 137355 & 55,72 & 1998 & 30 & 69 \\
\hline Vitória da Conquista (BA) & 262494 & 81,94 & 1998 & 30 & 39 \\
\hline Vitória (ES) & 292304 & 3284,31 & 1998 & 41 & 48 \\
\hline Aracaju (SE) & 461534 & 2549,91 & 1998 & 64 & 47 \\
\hline São Gonçalo (RJ) & 891119 & 3550,27 & 2001 & 179 & 68 \\
\hline Campinas (SP) & 969396 & 1217,83 & 2001 & 100 & 35 \\
\hline Goiânia (GO) & 1093007 & 1471,96 & 1998 & 75 & 23 \\
\hline Manaus (AM) & 1405835 & 123,23 & 1999 & 171 & 41 \\
\hline Brasília (DF) & 2051146 & 353,52 & 1997 & 68 & 11 \\
\hline
\end{tabular}

os profissionais das equipes de saúde da família com cobertura censitária em cinco municípios e amostras representativas em Manaus, Aracaju e Brasília, totalizando 2576 profissionais que preencheram questionários. Finalmente, foi feito um inquérito com famílias. Entrevistadores aplicaram um questionário em amostras por conglomerado de 240 famílias cadastradas em seis municípios, e de 100 famílias nos municípios de Vitória da Conquista e Goiânia (nos quais foram realizados estudos pilotos), totalizando 1640 famílias pesquisadas.

Em Campinas e São Gonçalo, não foram estudadas as equipes e as famílias, pois, no momento da pesquisa, o programa encontrava-se em fase inicial de implantação nesses municípios. $O$ trabalho de campo foi desenvolvido nos meses de março a julho de 2002, com exceção dos estudos pilotos, realizados no segundo semestre de 2001 (9). A partir dos resultados da pesquisa, foram selecionadas variáveis e construídos indicadores para o exame de possíveis mudanças do modelo assistencial introduzidas pelo PSF. Essas variáveis e indicadores são descritos e discutidos a seguir.

\section{O Programa de Saúde da Família como porta de entrada no sistema de saúde}

Um atributo fundamental da atenção primária é a sua definição como serviço de primeiro contato ou porta de entrada do sistema de saúde, com o papel de garantir atenção à maior parte das necessidades de saúde e filtrar o acesso aos outros níveis. $\mathrm{O}$ cumprimento dessa função pelo serviço de atenção primária implica acessibilidade (geográfica, tempo- ral e cultural), utilização do serviço por parte dos usuários a cada novo episódio e exigência e realização de encaminhamento dos profissionais de atenção primária para acesso à atenção especializada (4).

O primeiro requisito é que a unidade de saúde da família seja acessível à população adscrita, eliminando-se barreiras financeiras, geográficas, temporais e culturais, aspectos em geral contemplados pelo PSF. Não há barreiras financeiras para o acesso ao SUS, que é financiado por recursos fiscais de acesso universal, sem taxas de co-pagamento. Nas cidades estudadas, a grande maioria das famílias adscritas ao PSF (>90\%) afirmou conhecer o local da unidade de saúde da família, exceto em Goiânia (60\%). Entre as famílias que conheciam a localização da unidade de saúde da família, mais de $90 \%$ afirmaram ser fácil chegar ao local e se deslocavam a pé até a unidade (tabela 2). A acessibilidade temporal foi adequada somente em Campinas, onde as unidades básicas de saúde prestavam atendimento em três turnos e aos sábados. Nas outras cidades, o horário de funcionamento da unidade era das 8 às 17 horas, o que dificultava o acesso da população trabalhadora. A acessibilidade cultural no PSF é facilitada pela atuação do agente comunitário, elo mediador entre a comunidade e o serviço de saúde, na medida em que esse profissional é morador da comunidade de referência da unidade de saúde da família e, portanto, em geral pertence ao mesmo grupo social dos usuários; contudo, essa dimensão do acesso não foi objeto de avaliação específica no presente estudo.

A maioria das famílias entrevistadas (> 90\%) já havia utilizado alguma vez os serviços da equipe de saúde da família, e de 50 a 70\% haviam recebido 
TABELA 2. Acesso e utilização da unidade de saúde da família em oito centros urbanos, Brasil, 2002a

\begin{tabular}{|c|c|c|c|c|c|c|c|c|}
\hline Indicadores ${ }^{b}$ & $\begin{array}{l}\text { Camaragibe } \\
\qquad(\mathrm{PE})\end{array}$ & $\begin{array}{l}\text { Palmas } \\
\text { (TO) }\end{array}$ & $\begin{array}{l}\text { Vitória da } \\
\text { Conquista } \\
\text { (BA) }\end{array}$ & $\begin{array}{l}\text { Vitória } \\
\text { (ES) }\end{array}$ & $\begin{array}{l}\text { Aracaju } \\
\text { (SE) }\end{array}$ & $\begin{array}{l}\text { Goiânia } \\
\text { (GO) }\end{array}$ & $\begin{array}{l}\text { Manaus } \\
\text { (AM) }\end{array}$ & $\begin{array}{l}\text { Brasília } \\
\text { (DF) }\end{array}$ \\
\hline $\begin{array}{l}\text { Famílias, entre as que conhecem, que } \\
\text { afirmaram ser fácil chegar à USF }(\%)^{c}\end{array}$ & 95,7 & 95,7 & 92,4 & 93,6 & 95,1 & 94,1 & 97,1 & 91,6 \\
\hline ESF nos últimos 30 dias, inclusive de ACS (\%) & 48,7 & 67,1 & 82,0 & 57,1 & 72,9 & 62,7 & 65,1 & 48,1 \\
\hline \multicolumn{9}{|l|}{ Utilização de serviços pelas famílias cadastradas } \\
\hline Geralmente procuram a USF & 36,6 & 56,3 & 67,3 & 56,2 & 62,9 & 22,0 & 27,3 & 31,3 \\
\hline episódio de doença(\%) & 20,0 & 39,1 & 25,6 & 41,4 & 61,6 & 32,7 & 24,1 & 23,1 \\
\hline \multicolumn{9}{|l|}{$\begin{array}{l}\text { Procuraram pronto-socorro, hospital, centro de } \\
\text { saúde com serviço de urgência nos últimos }\end{array}$} \\
\hline 30 dias em episódio de doença (\%) ${ }^{\mathrm{e}}$ & 69,1 & 43,7 & 44,1 & 44,4 & 14,6 & 23,1 & 33,3 & 43,3 \\
\hline \multicolumn{9}{|c|}{$\begin{array}{l}\text { Fonte: Núcleo de Estudos Político-Sociais em Saúde, Departamento de Administração e Planejamento em Saúde, Escola Nacional de Saúde Pública, Fundação Oswaldo } \\
\text { Cruz. }\end{array}$} \\
\hline \multicolumn{9}{|c|}{$\begin{array}{l}\text { a São consideradas apenas oito cidades porque em duas o Programa de Saúde da Família encontrava-se em estágio inicial de implantação, não tendo sido realizados } \\
\text { inquéritos com profissionais e famílias nessas cidades. } \mathrm{AM}=\text { Amazonas; } \mathrm{BA}=\mathrm{Bahia} ; \mathrm{DF}=\text { Distrito Federal; } \mathrm{ES}=\mathrm{Espírito} \mathrm{Santo;} \mathrm{GO} \mathrm{=} \mathrm{Goiás;} \mathrm{PE} \mathrm{=} \mathrm{Pernambuco;} \\
\mathrm{SE}=\text { Sergipe; } \mathrm{TO}=\text { Tocantins. }\end{array}$} \\
\hline \multicolumn{9}{|c|}{ b ACS = agente comunitário de saúde; ESF = equipe de saúde da família; USF = unidade de saúde da família. } \\
\hline \multirow{2}{*}{\multicolumn{9}{|c|}{$\begin{array}{l}\text { c n (famílias que conhecem a USF) = } 214 \text { em Camaragibe, } 143 \text { em Palmas, } 86 \text { em Vitória da Conquista, } 158 \text { em Vitória, } 187 \text { em Aracaju, } 56 \text { em Goiânia, em } 235 \text { Ma- } \\
\text { naus e } 222 \text { em Brasília. } \\
\text { d n (famílias) }=100 \text { em Vitória da Conquista e } 100 \text { em Goiânia; nas outras cidades, } 240 \text { famílias (total } 1640 \text { famílias). }\end{array}$}} \\
\hline & & & & & & & & \\
\hline \multicolumn{9}{|c|}{$\begin{array}{l}\text { e (famílias que procuraram serviços de saúde nos últimos } 30 \text { dias em episódio de doença) = } 107 \text { em Camaragibe, } 86 \text { em Palmas, } 43 \text { em Vitória da Conquista, } 86 \text { em } \\
\text { Vitória, } 137 \text { em Aracaju, em } 52 \text { Goiânia, } 144 \text { em Manaus e } 106 \text { em Brasília. }\end{array}$} \\
\hline
\end{tabular}

algum atendimento no mês anterior à pesquisa. Para determinar se a unidade de saúde da família era o serviço de primeiro contato com o sistema de saúde, foram utilizados dois indicadores. O primeiro foi a proporção de famílias que afirmaram geralmente procurar a equipe de saúde da família quando precisavam de atendimento por profissional de saúde. Em quatro cidades, a metade ou mais das famílias informaram que geralmente procuravam a unidade de saúde da família. Goiânia, Manaus e Brasília, municípios em que o PSF foi concebido como programa paralelo, apresentaram as proporções mais baixas de procura habitual da saúde da família pela população adscrita (tabela 2). $\mathrm{Na}$ experiência de uso recente frente a episódio de doença, entretanto, em todos os municípios foram observadas menores proporções do que as proporções de famílias que procuraram a unidade de saúde da família em casos de mal-estar ou enfermidade ocorridos nos 30 dias anteriores à pesquisa. Embora a maior parte dos episódios não fosse grave, os serviços mais procurados em seis das oito cidades com famílias pesquisadas foram os de urgência (tabela 2). Outros serviços de atenção básica, como postos e centros de saúde, continuavam sendo procurados em Manaus (22\%), Brasília (19\%) e Goiânia (14\%), indicando a existência de paralelismo de redes básicas. Os serviços privados não integrantes do SUS foram procurados por uma parcela menor das famílias: entre 5 e 12\% (11).
A percepção dos profissionais das equipes de saúde quanto à porta de entrada foi, em geral, mais positiva do que a das famílias. Em Camaragibe, Aracaju e Palmas, mais de $80 \%$ dos profissionais de nível superior integrantes da equipe de saúde da família concordaram "muito" (dentre as opções "concorda muito", "concorda em parte" e "discorda") com a afirmativa de que a unidade de saúde da família se tornara a porta de entrada do sistema de atenção. Resultados muito inferiores à média foram observados em Brasília e Manaus, onde apenas a metade dos profissionais de nível superior concordou "muito" com a afirmativa (tabela 3).

Um atributo importante da constituição da atenção primária como serviço de primeiro contato é a exigência de encaminhamento pelo profissional de atenção primária para acesso aos serviços especializados. Essa função, contudo, não foi regulamentada no PSF, de forma que o seu exercício depende do grau de organização de cada sistema local. Em seis das 10 cidades estudadas, os gestores municipais concebiam o PSF como estratégia estruturante da rede de serviços, na qual a equipe de saúde da família deveria tornar-se a porta de entrada de sistema integrado. Em quatro dessas cidades (e em uma parcialmente), o acesso aos serviços especializados estava condicionado à referência pelo médico da equipe de saúde da família, podendo-se dizer que este exercia a função de porta de entrada. Em cinco cidades, as consultas com es- 
Temas de actualidad • Current topics

TABELA 3. Indicadores da unidade de saúde da família como serviço de primeiro contato em 10 centros urbanos, Brasil, 2002

\begin{tabular}{|c|c|c|c|c|}
\hline Municípioa & $\begin{array}{l}\text { Profissionais de nível } \\
\text { superior concordaram } \\
\text { "muito" que USF era } \\
\text { porta de entrada do } \\
\text { sistema de atençãob (\%) }\end{array}$ & $\begin{array}{l}\text { ESF era porta } \\
\text { de entrada } \\
\text { no sistema }\end{array}$ & $\begin{array}{l}\text { ESF agendava } \\
\text { especialistas }^{c}\end{array}$ & $\begin{array}{l}\text { Adscrição de clientela } \\
\text { na rede básica } \\
\text { tradicional }^{\mathrm{d}}\end{array}$ \\
\hline Camaragibe (PE) & 92,4 & Sim & Não & Quase toda a rede convertida para PSF \\
\hline Palmas (TO) & 82,7 & Sim & Sim & Toda a rede convertida para PSF \\
\hline Vitória da Conquista (BA) & 65,6 & Sim & Sim & Sim \\
\hline Vitória (ES) & 69,8 & Não & Sim & Sim \\
\hline Aracaju (SE) & 80,9 & Parcial & Sim & Quase toda a rede convertida para PSF \\
\hline São Gonçalo (RJ) & $\ldots$ & Não & Não & Não \\
\hline Campinas (SP) & $\ldots$ & Sim & Sim & Sim \\
\hline Goiânia (GO) & 67,0 & Não & Não & Não \\
\hline Manaus (AM) & 55,4 & Não & Não & Não \\
\hline Brasília (DF) & 51,6 & Não & Não & Sim \\
\hline \multicolumn{5}{|c|}{$\begin{array}{l}\text { Fonte: Núcleo de Estudos Político-Sociais em Saúde, Departamento de Administração e Planejamento em Saúde, Escola Nacional de Saúde Pública, Fundação Os- } \\
\text { waldo Cruz. } \\
\text { a AM = Amazonas; BA = Bahia; DF = Distrito Federal; ES = Espírito Santo; GO = Goiás; PE = Pernambuco; RJ = Rio de Janeiro; SE = Sergipe; SP = São Paulo; TO = } \\
\text { Tocantins. } \\
\text { b n (profissionais de nível superior) = } 53 \text { em Camaragibe, } 52 \text { em Palmas, } 64 \text { em Vitória da Conquista, } 63 \text { em Vitória, } 94 \text { em Aracaju, } 94 \text { em Goiânia, } 60 \text { em Manaus e } \\
74 \text { em Brasília. As opções de resposta incluíam "concorda muito", "concorda em parte" e "discorda". No momento do estudo, o Programa de Saúde da Família } \\
\text { encontrava-se em início de implantação em São Gonçalo e Campinas, não tendo sido realizado inquérito com profissionais nessas cidades. USF = unidade de saúde } \\
\text { da família. } \\
\text { c ESF = equipe de saúde da família. } \\
\text { d PSF = Programa de Saúde da Família. }\end{array}$} \\
\hline
\end{tabular}

pecialistas eram marcadas pela equipe de saúde da família, indicando forte preocupação com a garantia da continuidade do cuidado (tabela 3).

\section{Integração do Programa de Saúde da Família à rede assistencial}

A integração das unidades de saúde da família à rede assistencial é fundamental para garantir uma oferta abrangente de serviços e para coordenar as diversas ações requeridas para resolver as necessidades menos freqüentes e mais complexas. A coordenação implica a capacidade de garantir a continuidade da atenção ao longo da rede de serviços por meio de mecanismos de integração, tecnologias de gestão clínica e instrumentos para a comunicação entre profissionais de diferentes serviços (4). A integração do PSF com os demais níveis de complexidade foi avaliada por meio da presença e uso de mecanismos de referência e contra-referência pelos profissionais das equipes de saúde da família, possibilidades de agendamento pelos profissionais e experiência das famílias quanto à realização de exames.

$\mathrm{Na}$ maior parte dos municípios estudados, havia alguma forma de regulação para a referência, contudo somente em quatro deles estavam estruturadas centrais de marcação de consultas especiali- zadas (uma delas não informatizada). Em três cidades funcionavam centrais de regulação de internações sob o comando municipal. Na ausência de centrais estruturadas, alguns municípios estabeleceram cotas físicas ou financeiras ${ }^{4}$ de consultas especializadas ou exames diagnósticos por unidade de saúde, sendo sua existência confirmada pelo uso das mesmas pelos profissionais das equipes de saúde da família para encaminhamentos de pacientes para consultas especializadas (11).

A existência de mecanismos de integração, contudo, não era suficiente para garantir um sistema de referência em pleno funcionamento. Em sete das cidades estudadas, menos de $15 \%$ dos profissionais das equipes concordaram "muito" com a afirmativa de que o Saúde da Família conta com um sistema de referência e contra-referência. A exceção foi Vitória da Conquista, evidenciando um melhor funcionamento do sistema naquela cidade.

$\mathrm{Na}$ maioria dos municípios, os gestores reconheciam uma importante dificuldade para garantir

\footnotetext{
4 Cotas financeiras são montantes de recursos disponibilizados semanalmente ou mensalmente por unidades básicas de saúde para a realização de exames em laboratórios privados contratados; cotas físicas são quantidades definidas de consultas com determinados especialistas, disponibilizadas periodicamente pelas secretarias municipais de saúde para que cada unidade de saúde da família agende seus pacientes encaminhados, garantindo uma distribuição mais eqüitativa entre as unidades.
} 
a atenção secundária; no entanto, não havia monitoramento que permitisse conhecer a magnitude das filas e os tempos de espera. As famílias percebiam essa dificuldade de modo mais intenso do que os profissionais das equipes de saúde da família. Em todas as cidades estudadas, mais da metade dos médicos das equipes de saúde da família informou conseguir agendar serviços ambulatoriais de média complexidade sempre ou na maioria das vezes. As maiores dificuldades para o agendamento de serviços de média complexidade foram observadas em Brasília, Aracaju e Palmas. A realização de exames diagnósticos foi uma dificuldade importante para as famílias pesquisadas: em cinco das oito cidades nas quais foi realizado inquérito com famílias, mais da metade das famílias informou não conseguir realizar todos os exames solicitados.

A comunicação com troca de informações entre profissionais é essencial para que o generalista da equipe de saúde da família possa exercer sua função de coordenador dos cuidados ao paciente e garantir a continuidade do contato. Embora existissem formulários para a transferência de informações entre médicos do PSF e especialistas, na maioria das cidades seu uso não era freqüente (tabela 4). A insuficiente realização da contrareferência foi uma dificuldade reiterada pelos ges- tores na maior parte dos municípios estudados, o que foi confirmado pelos profissionais e é bem ilustrado pelas palavras de uma experiente gestora do programa: "Até hoje, eu só conheço um médico nosso que recebeu uma contra-referência através do mecanismo formal. Ele comemorou e até colocou no quadro de avisos".

Outro mecanismo de coordenação e de redução da variedade das condutas é o uso de protocolos clínicos. Em algumas cidades, o PSF utiliza manuais com protocolos clínicos elaborados pelo Ministério da Saúde para acompanhamento dos grupos prioritários, como os protocolos de Assistência Integrada às Doenças Prevalecentes na Infância, hipertensão arterial, diabetes, planejamento familiar, saúde da mulher, pré-natal e doenças sexualmente transmissíveis/AIDS. No entanto, em apenas três cidades a elaboração e utilização de protocolos era uma preocupação dos gestores locais (Vitória da Conquista, Vitória e Aracaju).

\section{Incorporação de novas práticas assistenciais}

A conversão do modelo de atenção básica à saúde operacionaliza-se por meio da organização do trabalho em equipe, com a substituição de práti-

TABELA 4. Indicadores de integração e coordenação do Programa de Saúde da Família em oito centros urbanos, Brasil, $2002^{\mathrm{a}}$

\begin{tabular}{|c|c|c|c|c|c|}
\hline \multirow[b]{3}{*}{ Municípiod } & \multirow{2}{*}{\multicolumn{2}{|c|}{$\begin{array}{l}\text { Porcentagem de médicos que } \\
\text { agendamb }\end{array}$}} & \multirow{3}{*}{$\begin{array}{l}\text { Porcentagem de } \\
\text { famílias que } \\
\text { informaram conseguir } \\
\text { realizar todos os } \\
\text { exames solicitados }\end{array}$} & \multicolumn{2}{|c|}{$\begin{array}{l}\text { Porcentagem de profissionais } \\
\text { de nível superiorc }\end{array}$} \\
\hline & & & & \multirow{2}{*}{$\begin{array}{c}\text { Concordam } \\
\text { "muito" que ESF } \\
\text { conta com sistema } \\
\text { de referência }\end{array}$} & \multirow{2}{*}{$\begin{array}{l}\text { Nunca recebem } \\
\text { contra- } \\
\text { referência }\end{array}$} \\
\hline & $\begin{array}{l}\text { Consulta com } \\
\text { especialista }\end{array}$ & Maternidade & & & \\
\hline Camaragibe (PE) & 81,8 & 54,5 & 31,7 & 3,8 & 34,0 \\
\hline Palmas (TO) & 58,3 & 83,3 & 48,6 & 11,5 & 44,2 \\
\hline Vitória da Conquista (BA) & 62,5 & $\ldots^{g}$ & 69,9 & 54,7 & $\ldots{ }^{g}$ \\
\hline Vitória (ES) & 70,9 & 58,0 & 59,2 & 1,6 & 43,0 \\
\hline Aracaju (SE) & 57,6 & 69,7 & 51,7 & 13,8 & 53,0 \\
\hline Goiânia (GO) & 62,2 & $\ldots^{g}$ & 30,8 & 10,6 & $\ldots{ }^{g}$ \\
\hline Manaus (AM) & 75,0 & 64,3 & 40,4 & 12,5 & 34,0 \\
\hline Brasília (DF) & 55,5 & 66,7 & 35,4 & 13,5 & 39,2 \\
\hline \multicolumn{6}{|c|}{$\begin{array}{l}\text { Fonte: Núcleo de Estudos Político-Sociais em Saúde, Departamento de Administração e Planejamento em Saúde, Escola Nacional de Saúde Pública, Fundação Os- } \\
\text { waldo Cruz. } \\
\text { a São consideradas apenas oito cidades porque em duas o Programa de Saúde da Família encontrava-se em estágio inicial de implantação, não tendo sido realizados } \\
\text { inquéritos com profissionais e famílias nessas cidades. }\end{array}$} \\
\hline \multicolumn{6}{|c|}{$\begin{array}{l}\text { b Sempre/na maioria das vezes. Total de médicos pesquisados: } 22 \text { em Camaragibe, } 24 \text { em Palmas, } 24 \text { em Vitória da Conquista, } 31 \text { em Vitória, } 33 \text { em Aracaju, } 45 \text { em } \\
\text { Goiânia, } 28 \text { em Manaus e } 27 \text { em Brasília. }\end{array}$} \\
\hline \multicolumn{6}{|c|}{ 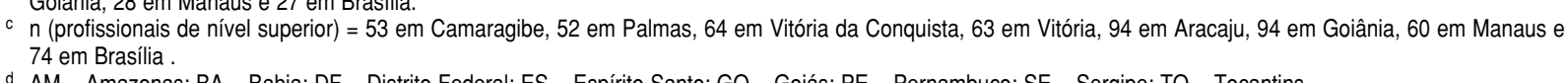 } \\
\hline \multirow{2}{*}{\multicolumn{6}{|c|}{$\begin{array}{l}\text { AM = Amazonas; } B A=\text { Bahla; } D F=\text { Distrito Federal; } E S=\text { Espirito Santo; } G O=\text { Golas; } P E=\text { Pernambuco; } S E=\text { Sergipe; } 10=10 \text { lo } \\
\text { e As opções de resposta incluíam "concorda muito", "concorda em parte" e "discorda". ESF = equipe de saúde da família. }\end{array}$}} \\
\hline & & & & & \\
\hline \multicolumn{6}{|c|}{ f A resposta inclui a opção "recebem informação verbal pelo paciente". } \\
\hline g Informação não disponível & & & & 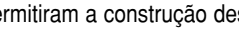 & \\
\hline
\end{tabular}


cas convencionais de assistência e a incorporação de novas práticas voltadas para a família e a comunidade, com o objetivo de influenciar os determinantes sociais do processo saúde-enfermidade (9).

Um processo de trabalho apoiado em uma equipe multiprofissional apta a oferecer atenção integral e a sustentar o enfoque social ampliado da estratégia de saúde da família, com claros objetivos de inclusão social, exige qualificação dos profissionais inseridos e adesão ao programa por parte desses profissionais. $\mathrm{O}$ estudo do perfil dos profissionais das equipes de saúde da família revelou predomínio do gênero feminino, especialmente entre auxiliares de enfermagem e agentes comunitários (> 75\%), equipes jovens e um bom nível de escolaridade. Os agentes comunitários superaram o grau mínimo exigido pelo programa, "saber ler e escrever". Em cinco municípios, mais de 60\% dos agentes tinham segundo grau completo. Um percentual pequeno (até 15\%) de agentes tinha curso técnico em saúde, a não ser em Goiânia $(68 \%)$.

Contudo, a formação específica dos profissionais de nível superior das equipes para atuação em atenção primária foi baixa na maioria das cidades. Em seis municípios, o percentual de profissionais de nível superior com especialização nas áreas de saúde pública e coletiva ou com formação voltada para a saúde da família foi inferior a 30\% (9). Entre os fatores que motivaram a escolha dos profissionais pela atuação no PSF, destacaram-se trabalhar junto a comunidades pobres e a possibilidade do PSF de reorganizar a atenção no âmbito municipal, o que aponta para o reconhecimento da dimensão ideológica do programa quanto à transformação do modelo assistencial. Os menores índices de adesão, por esses motivos, foram observados entre os profissionais de Manaus e Brasília. Entre os agentes comunitários, a superação do desemprego foi o principal motivo para a escolha dessa atividade (mais de $60 \%$ dos agentes em quatro cidades).

A análise da introdução de novas práticas nos serviços de saúde está relacionada à abrangência (integralidade) da atenção prestada, um dos atributos da atenção primária em saúde (4), e ao direcionamento da atuação da equipe de saúde da família para a família e a comunidade. Voltar-se para a comunidade implica identificar suas necessidades de saúde, reconhecer as fases do ciclo de vida e diferenças de exposição aos fatores de risco entre os grupos populacionais, o que permitiria poder responder com competência às necessidades das famílias adscritas. No presente estudo, avaliou-se a integralidade da atenção pela realização, na semana anterior à pesquisa, de atividades preventivas e assistenciais selecionadas e pelo envolvimento da equipe de saúde da família em atividades extramu- ros e intersetoriais direcionadas às famílias e à comunidade, relacionadas aos condicionantes do estado de saúde da população, como, por exemplo, busca de solução para problemas de coleta de lixo, abastecimento insuficiente de água, ausência de esgotamento sanitário e crianças fora da escola, por meio de contatos com os setores responsáveis e mobilização das comunidades.

Quanto às atividades dos profissionais de nível superior que envolvem a promoção da saúde e a prevenção das doenças, embora a maioria tenha informado desenvolvê-las, observou-se, entre os médicos, uma baixa proporção de profissionais que informaram ter realizado atividades de educação em saúde na semana anterior à pesquisa, variando de 22\% em Brasília a 53\% em Aracaju. Entre os enfermeiros, a proporção foi mais elevada, tendo cerca da metade ou mais dos profissionais realizado atividades de educação na semana anterior em todas as cidades estudadas, exceto em Manaus (38\%) (tabela 5).

Entre as novas práticas incorporadas pelas equipes de saúde da família destacam-se as atividades de grupo com pacientes em atendimento na unidade de saúde da família, realizadas por proporções semelhantes de médicos e enfermeiros (de 26 a 54\%) na semana anterior à pesquisa. Na área curativo-assistencial, analisou-se a consulta individual à população cadastrada (como indicador de assistência oportuna) e a consulta individual aos grupos prioritários, indicando a incorporação da perspectiva de estruturação da demanda preconizada no programa. Na semana anterior à pesquisa, os médicos das equipes de saúde da família desenvolveram com maior freqüência consultas para a população cadastrada do que para grupos prioritários. Consultas individuais aos grupos prioritários foram realizadas com maior freqüência pelos enfermeiros (tabela 5).

As atividades extramuros aqui selecionadas foram a visita domiciliar e a reunião com a comunidade realizadas pelos profissionais de nível superior na semana anterior à pesquisa. A grande maioria dos profissionais de nível superior (de 70 a 90\%) realizou visitas domiciliares nos municípios estudados para prover atenção clínica ou promover a saúde, indicando que esse tipo de atuação extramuros foi incorporado à rotina dos profissionais das equipes de saúde da família (tabela 6). Observaramse, todavia, baixas proporções de profissionais de nível superior que realizaram reunião com a comunidade, ainda que mais da metade (66 a 96\%) informasse realizá-las às vezes (tabela 6), com destaque para Camaragibe (96\%) (9).

Para os agentes comunitários, as visitas domiciliares são atribuição precípua. A proporção de agentes que fizeram visita domiciliar na semana an- 
TABELA 5. Distribuição percentual de médicos e enfermeiros das equipes de saúde da família que realizaram atividades de educação em saúde e assistenciais na semana anterior à pesquisa em seis centros urbanos, Brasil, 2002a

\begin{tabular}{|c|c|c|c|c|c|c|c|c|}
\hline \multirow[b]{3}{*}{ Municípiod } & \multicolumn{4}{|c|}{ Educação em saúde b } & \multicolumn{4}{|c|}{ Consultas $^{b}$} \\
\hline & \multicolumn{2}{|c|}{ Atividades individuais } & \multicolumn{2}{|c|}{ Atividades de grupo } & \multicolumn{2}{|c|}{ População adscrita } & \multicolumn{2}{|c|}{ Grupos prioritários $^{c}$} \\
\hline & Médico & Enfermeiro & Médico & Enfermeiro & Médico & Enfermeiro & Médico & Enfermeiro \\
\hline Camaragibe (PE) & 22,7 & 48,1 & 45,5 & 37,0 & 90,9 & 88,9 & 36,4 & 33,3 \\
\hline Palmas (TO) & 29,2 & 67,9 & 54,2 & 39,3 & 54,2 & 75,0 & 33,3 & 53,6 \\
\hline Vitória (ES) & 22,6 & 65,6 & 25,8 & 31,3 & 77,4 & 46,9 & 32,3 & 50,0 \\
\hline Aracaju (SE) & 53,3 & 47,1 & 36,4 & 47,1 & 69,7 & 76,5 & 42,4 & 50,0 \\
\hline Manaus (AM) & 25,0 & 38,1 & 39,3 & 38,1 & 67,9 & 57,1 & 42,9 & 33,3 \\
\hline Brasília (DF) & 22,2 & 55,6 & 40,7 & 44,4 & 92,6 & 92,6 & 29,6 & 33,3 \\
\hline
\end{tabular}

Fonte: Núcleo de Estudos Político-Sociais em Saúde, Departamento de Administração e Planejamento em Saúde, Escola Nacional de Saúde Pública, Fundação Oswaldo Cruz.

a Nos estudos pilotos em Vitória da Conquista e Goiânia, os dados coletados não permitiram a construção desses indicadores, por isso nesta tabela são apresentados dados de apenas seis municípios.

b $\mathrm{n}$ (médicos) = 22 em Camaragibe, 24 em Palmas, 31 em Vitória, 33 em Aracaju, 28 em Manaus e 27 em Brasília. $n$ (enfermeiros) = 27 em Camaragibe, 28 em Palmas, 32 em Vitória, 34 em Aracaju, 21 em Manaus e 27 em Brasília.

c Grupos prioritários: crianças menores de 5 anos, gestantes, saúde da mulher, portadores de hipertensão arterial, portadores de diabetes, portadores de tuberculose e portadores de hanseníase.

d $\mathrm{AM}=$ Amazonas; DF = Distrito Federal; ES = Espírito Santo; PE = Pernambuco; SE = Sergipe; TO = Tocantins.

TABELA 6. Distribuição percentual de profissionais das equipes de saúde da família que realizaram atividades extramuros e intersetoriais em seis centros urbanos, Brasil, 2002

\begin{tabular}{|c|c|c|c|c|c|c|}
\hline \multirow[b]{2}{*}{ Municípiob } & \multicolumn{2}{|c|}{$\begin{array}{c}\text { Realização } \\
\text { de visita } \\
\text { domiciliar na } \\
\text { semana anterior }\end{array}$} & \multirow{2}{*}{$\begin{array}{c}\text { Ação dirigida } \\
\text { à comunidade } \\
\text { na semana } \\
\text { anterior } \\
\text { ACS }\end{array}$} & \multirow{2}{*}{$\begin{array}{c}\text { Reunião com } \\
\text { a comunidade } \\
\text { na semana } \\
\text { anterior } \\
\text { NS }\end{array}$} & \multirow{2}{*}{$\begin{array}{c}\text { Atuação } \\
\text { intersetorial } \\
\text { nos } 6 \text { meses } \\
\text { anteriores } \\
\text { ACS }\end{array}$} & \multirow{2}{*}{$\begin{array}{c}\text { Realização de contatos } \\
\text { com órgãos } \\
\text { governamentais na } \\
\text { semana anterior } \\
\text { NS }\end{array}$} \\
\hline & ACS & NS & & & & \\
\hline Camaragibe (PE) & 99,5 & 96,2 & 68,8 & 9,4 & 60,7 & 1,9 \\
\hline Palmas (TO) & 99,4 & 94,2 & 86,5 & 9,6 & 81,6 & 9,6 \\
\hline Vitória (ES) & 99,5 & 77,8 & 56,9 & 6,3 & 78,3 & 4,8 \\
\hline Aracaju (SE) & 99,4 & 71,3 & 55,1 & 11,7 & 40,3 & 0,0 \\
\hline Manaus (AM) & 91,6 & 82,1 & 60,2 & 10,7 & 54,4 & 5,4 \\
\hline Brasília (DF) & 98,3 & 87,8 & 73,9 & 14,9 & 59,1 & 4,1 \\
\hline
\end{tabular}

Fonte: Núcleo de Estudos Político-Sociais em Saúde, Departamento de Administração e Planejamento em Saúde, Escola Nacional de Saúde Pública, Fundação Oswaldo Cruz.

a ACS = agente comunitário de saúde; NS = profissional de nível superior. $\mathrm{n}$ (profissionais de nível superior) = 53 em Camaragibe, 52 em Palmas, 63 em Vitória, 94 em Aracaju, 60 em Manaus e 74 em Brasília. n (agentes comunitários) = 186 em Camaragibe, 163 em Palmas, 202 em Vitória, 176 em Aracaju, 261 em Manaus e 115 em Brasília.

b $\mathrm{AM}=$ Amazonas; DF = Distrito Federal; ES = Espírito Santo; PE = Pernambuco; SE = Sergipe; TO = Tocantins.

terior à coleta de dados foi superior a 90\% em todos os municípios. A alta adesão dos agentes comunitários à realização de ações voltadas para a comunidade (tabela 6) expressa a força de um novo perfil de atuação, com destaque para Palmas (87\%) e Brasília (74\%).

Em termos da incorporação de ações intersetoriais às práticas profissionais, foram investigadas as atividades junto a outros órgãos de políticas públicas pelos profissionais das equipes de saúde da família, a percepção dos profissionais e a avaliação das famílias quanto ao conhecimento da equipe acerca dos problemas de saúde da comunidade. Embora entre um terço e metade dos profissionais de nível superior da equipe de saúde da família tenha concordado com a afirmativa de que "a equipe de saúde da família desenvolve atividades fora do setor saúde voltadas para a solução de problemas das comunidades" (9), as respostas positivas quanto à realização de contatos com outros 
órgãos governamentais na semana anterior à pesquisa apresentaram proporções muito baixas, sendo nulas em Aracaju e alcançando 9,6\% em Palmas (tabela 6). A atuação dos agentes comunitários, por outro lado, esteve mais voltada para a articulação intersetorial: mais da metade ( 54 a $82 \%$ ) havia mantido contato com outros setores nos últimos 6 meses, a não ser em Aracaju (tabela 6).

Apesar de a participação dos profissionais de nível superior em atividades extra-setoriais não ter sido elevada, a maioria ( $>66 \%$ ) afirmou que a equipe de saúde da família deve relacionar-se com outros órgãos, organizações não-governamentais e organizações da sociedade civil para resolver os problemas da comunidade. Coerente com a baixa atuação informada, apenas um terço ou menos desses profissionais considerou muito satisfatória a articulação da equipe de saúde da família com outros setores sociais atuantes na área (tabela 7). Os agentes comunitários, em razão de sua atuação, têm percepções mais positivas quanto à ação intersetorial das equipes de saúde da família. Camaragibe, Goiâ-

TABELA 7. Percepção da intersetorialidade por profissionais das equipes de saúde da família e avaliação sobre conhecimento dos problemas da comunidade em oito centros urbanos, Brasil, 2002 ${ }^{\mathrm{a}}$

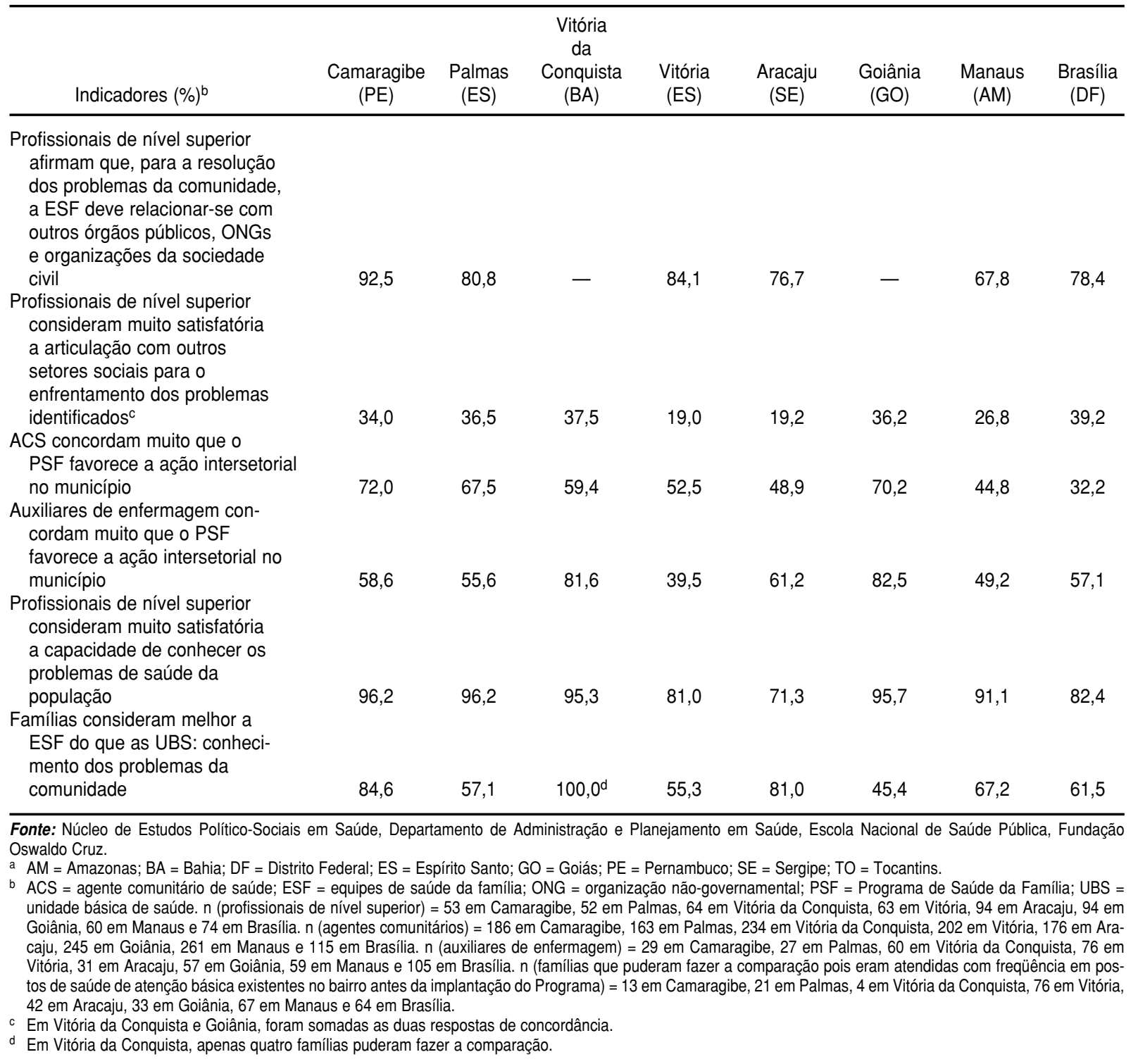


nia e Palmas apresentaram as maiores proporções de agentes que concordaram "muito" com a afirmativa de que o PSF favorece a ação intersetorial no município. Entre os auxiliares de enfermagem, elevadas proporções também concordaram "muito" que o PSF favorece a ação intersetorial no município.

Para responder com competência às necessidades de saúde da população, os profissionais devem conhecê-las e estabelecer prioridades. Os profissionais de nível superior das equipes de saúde da família, em sua maioria $(\geq 70 \%)$, consideraram muito satisfatório o conhecimento das equipes do PSF em relação aos principais problemas de saúde da população, avaliação em geral corroborada pela opinião das famílias, que, em sua maioria, consideraram a equipe de saúde da família melhor nesse aspecto do que os profissionais das unidades básicas de saúde prévias (exceto em Goiânia).

As principais responsabilidades da atenção básica no Brasil estão reunidas em sete grupos de ações: saúde da criança, saúde da mulher, controle da hipertensão, controle do diabetes, controle da tuberculose, eliminação da hanseníase e saúde bucal. Fechando essa dimensão de análise, buscou-se verificar se as equipes estabeleciam algum tipo de prioridade para o atendimento da população cadastrada, e levantou-se, junto aos que responderam afirmativamente, a hierarquia estabelecida pelas equipes. Em todos os municípios estudados, uma grande proporção dos integrantes das equipes de saúde da família (83 a 99\%) afirmou que a equipe estabelece prioridades para atendimento. Quando lhes foi solicitado que assinalassem as ações prioritárias desenvolvidas a partir de uma lista contendo 13 itens, tanto os profissionais de nível superior quanto os auxiliares de enfermagem e os agentes comunitários indicaram, em todos os municípios, que era conferida prioridade, no atendimento às famílias, para o acompanhamento da hipertensão arterial (82 a 100\%), da criança (66 a 100\%), das ações de pré-natal (83 a 97\%) e do diabetes (78 a 98\%) (9).

$\mathrm{O}$ atendimento a esses grupos foi também investigado junto às famílias, cotejando estas respostas às dos profissionais. No conjunto de grandes centros urbanos, observou-se elevado percentual de famílias que informaram a existência de portadores de doenças crônicas-mais de 50 e até 67\% (Brasília) dos domicílios pesquisados, exceto em Palmas. A hipertensão arterial e o diabetes foram as doenças crônicas auto-referidas com maior freqüência (tabela 8). A proporção de gestantes foi reduzida (4 a $6 \%$ dos domicílios). As crianças menores de 2 anos estavam presentes em cerca de $20 \%$ dos domicílios pesquisados, com proporções mais baixas em Manaus e mais altas em Aracaju.

Destacam-se, neste artigo, alguns resultados relativos ao acompanhamento dos portadores de diabetes e hipertensão. Em todos os seis municípios pesquisados quanto a essas variáveis, as equipes de saúde da família realizavam o acompanhamento de

TABELA 8. Distribuição percentual de famílias usuárias do Programa de Saúde da Família com portadores de diabetes e hipertensão arterial e atendimentos recebidos em seis centros urbanos, Brasil, 2002

\begin{tabular}{|c|c|c|c|c|c|c|}
\hline \multirow[b]{2}{*}{ Município ${ }^{e}$} & \multicolumn{2}{|c|}{$\begin{array}{l}\text { Famílias com } \\
\text { diabéticos }^{\mathrm{b}}\end{array}$} & \multicolumn{2}{|c|}{$\begin{array}{l}\text { Famílias com } \\
\text { hipertensos }^{c}\end{array}$} & \multicolumn{2}{|c|}{$\begin{array}{l}\text { Indivíduos acompanhados } \\
\text { pela ESFd }\end{array}$} \\
\hline & $\%$ & $\begin{array}{l}\text { Sem } \\
\text { tratamento }\end{array}$ & $\%$ & $\begin{array}{l}\text { Sem } \\
\text { tratamento }\end{array}$ & $\begin{array}{c}\text { Diabéticos } \\
\text { E }\end{array}$ & $\begin{array}{l}\text { Hipertensos } \\
\text { F }\end{array}$ \\
\hline Camaragibe (PE) & 12,9 & 6,5 & 42,3 & 11,8 & 81,3 & 75,4 \\
\hline Palmas (TO) & 3,8 & 30,8 & 15,4 & 12,8 & 53,8 & 66,7 \\
\hline Vitória (ES) & 9,2 & 14,8 & 40,4 & 7,0 & 62,1 & 71,2 \\
\hline Aracaju (SE) & 11,6 & 7,1 & 30,8 & 20,3 & 54,8 & 54,9 \\
\hline Manaus (AM) & 12,9 & 29,0 & 27,8 & 20,9 & 50,0 & 63,0 \\
\hline Brasília (DF) & 8,3 & 25,8 & 30,0 & 12,1 & 38,7 & 57,7 \\
\hline
\end{tabular}

Fonte: Núcleo de Estudos Político-Sociais em Saúde, Departamento de Administração e Planejamento em Saúde, Escola Nacional de Saúde Pública, Fundação Oswaldo Cruz.

a $n=240$ famílias em cada cidade ( $n$ total 1440 ). Nos estudos pilotos em Vitória da Conquista e Goiânia, os dados coletados não permitiram a construção desses indicadores; por isso, nesta tabela são apresentados dados de apenas seis municípios. ESF = equipe de saúde da família.

b $n$ (famílias com diabéticos) = 29 em Camaragibe, 13 em Palmas, 23 em Vitória, 28 em Aracaju, 22 em Manaus e 31 em Brasília.

c $\mathrm{n}$ (famílias com hipertensos) = 90 em Camaragibe, 47 em Palmas, 106 em Vitória, 74 em Aracaju, 52 em Manaus e 99 em Brasília.

d $n$ (indivíduos diabéticos) = 32 em Camaragibe, 13 em Palmas, 29 em Vitória, 31 em Aracaju, 36 em Manaus e 31 em Brasília. $n$ (indivíduos hipertensos) = 118 em Camaragibe, 54 em Palmas, 146 em Vitória, 91 em Aracaju, 81 em Manaus, 123 em Brasília.

e $\mathrm{AM}=$ Amazonas; $\mathrm{DF}=$ Distrito Federal; ES = Espírito Santo; PE = Pernambuco; SE = Sergipe; TO = Tocantins. 
saúde de metade ou mais do total de hipertensos e diabéticos, exceto em Brasília; entretanto, parte dos doentes auto-referidos permanecia sem tratamento. A proporção de famílias que informaram que os diabéticos não recebiam qualquer acompanhamento de saúde foi mais elevada em Palmas, Manaus e Brasília, verificando-se em Camaragibe o menor percentual de famílias em que os portadores de diabetes não estavam sob acompanhamento (tabela 8). Em famílias nas quais havia hipertensos, de 7 a 20\% informaram que os doentes não recebiam acompanhamento de saúde.

\section{CONSIDERAÇÕES FINAIS}

Após uma década do início da implantação do PSF no Brasil, observa-se um processo ainda em curso de mudança incremental do modelo assistencial da atenção básica. O estudo da implementação do PSF revela avanços na incorporação de novas práticas profissionais, na atenção primária e na criação de vínculos entre a equipe de saúde da família e as famílias adscritas. No entanto, sua expansão nos grandes centros urbanos enfrenta uma série de obstáculos.

A garantia da integralidade permanece como um importante nó crítico a ser equacionado. Nos municípios estudados, observou-se a ampliação da pressão da demanda por atenção secundária com a extensão de cobertura de atenção básica. Embora na metade das cidades a perspectiva dos gestores fosse a de que as equipes de saúde da família conformassem a porta de entrada do sistema de saúde, e de que a instauração de mecanismos de referência garantisse o atendimento integral aos munícipes, a unidade de saúde da família foi o serviço de procura regular para mais de $50 \%$ da população adscrita apenas em quatro dos oito municípios. Somente em quatro cidades o acesso aos serviços especializados estava condicionado à referência do médico da equipe de saúde da família. Uma baixa proporção de famílias havia procurado a unidade de saúde da família em episódio de doença ocorrido nos 30 dias anteriores à pesquisa. Embora a maior parte dos episódios não fosse grave, os serviços mais procurados em seis das oito cidades pesquisadas foram os de urgência; outros serviços de atenção básica, como postos e centros de saúde, continuavam sendo procurados em três cidades, indicando a sobreposição de redes básicas e a implantação do PSF como um programa paralelo.

$\mathrm{O}$ acesso à atenção secundária permaneceu difícil. Afora insuficiente disponibilidade de recursos financeiros, um importante limite à concretização, na prática, da conformação do PSF como porta de entrada foi a carência da oferta de serviços públicos municipais de média e alta complexidade, aliada, em algumas cidades, a uma descentralização incompleta, isto é, à sua condição de habilitação para a gestão do sistema de saúde junto ao Ministério da Saúde apenas no primeiro nível (Plena de Atenção Básica), com impossibilidade dos municípios de gerenciar toda a rede assistencial do SUS, e não apenas a rede básica, em seu território.

A referência era pouco estruturada, sendo grande a dificuldade para garantir o atendimento nas especialidades médicas e exames de apoio diagnóstico de média complexidade. A maioria das unidades de saúde da família não possui equipamentos de informática (12) e telefonia, muitas vezes valendo-se dos celulares e dos contatos dos próprios profissionais para conseguir o atendimento especializado. Na maior parte dos municípios estudados, havia alguma forma de regulação para a referência. Contudo, os mecanismos de integração não eram suficientes para garantir um sistema de referência e contra-referência que possibilitasse a coordenação do cuidado. Esse foi um dos atributos da atenção primária menos desenvolvidos nas experiências estudadas, permanecendo como grande desafio do PSF a melhora da comunicação entre profissionais da atenção básica e especialistas e serviços de pronto-atendimento e emergência. São profissionais com experiências e formações distintas, o que exige a criação de estratégias de comunicação e de ampliação da confiança e da credibilidade do profissional de atenção primária para que o especialista promova o retorno do paciente ao generalista, de forma a garantir a continuidade do tratamento pelo profissional mais adequado.

Outra importante dificuldade foi a substituição de práticas tradicionais e a adequada articulação entre as atividades clínicas e de saúde coletiva. A programação de atividades para grupos de risco e a oferta organizada confrontam-se com as demandas individuais por assistência, o que dificulta o acesso e o estabelecimento da unidade de saúde da família como porta de entrada ao sistema. Novas práticas voltadas para a família e a comunidade, com o objetivo de influenciar os determinantes sociais do processo saúde-enfermidade, foram incorporadas pelas equipes, ainda que parcialmente. Entre as novas práticas incorporadas destacam-se as atividades de grupo com pacientes em atendimento na unidade de saúde da família e as atividades extramuros, sobretudo as visitas domiciliares e as reuniões com a comunidade realizadas tanto por agentes comunitários quanto por profissionais de nível superior, embora em menor proporção. A atuação na comunidade e intersetorial foi atribuição principalmente dos agentes, o que su- 
gere ser a sua inclusão na equipe um componente fundamental para permitir a mudança do modelo de atenção básica. Esses integrantes da equipe de saúde da família, mais próximos da comunidade, não são profissionais de saúde no sentido tradicional, e podem vir a atuar como verdadeiros agentes de saúde coletiva. A intensificação da atuação intersetorial da equipe de saúde da família, contudo, parece estar condicionada a uma nova postura governamental, na qual o executivo municipal articularia as intervenções dos diversos setores para a solução dos problemas.

A estratégia de saúde da família, intensiva em força de trabalho, ainda encontra obstáculos na incorporação de profissionais relativos à inserção e ao desenvolvimento de recursos humanos. Os mecanismos de inserção dos profissionais das equipes de saúde por meio de contratos temporários e vínculos precários, na maior parte das cidades estudadas, podem afetar a sustentabilidade do programa. Segundo levantamento realizado pelo Ministério da Saúde, menos de um terço dos médicos e dos enfermeiros tinha vínculo contratual não precário em 2001 e 2002 (13). Vínculos empregatícios estáveis e legalmente protegidos favorecem a adesão de profissionais e a formação de laços com as comunidades. Aprimorar o processo de capacitação dos profissionais para suas novas funções é outro desafio a ser enfrentado, garantindo que todos os profissionais realizem treinamentos que superem a fragmentação programática dos conhecimentos, articulando, na capacitação permanente, aspectos técnico-científicos mais gerais com a especificidade dos condicionantes locais e com estratégias de humanização do atendimento.

Em síntese, pode-se afirmar que não há uniformidade quanto às modalidades implementadas do PSF. Em quatro casos, constituiu-se em um programa paralelo, com superposição de redes assistenciais básicas, correspondendo às críticas de focalização e seletividade com cesta mínima "promocional", dirigindo-se apenas a grupos populacionais muito pobres e realizando algumas ações educativas e preventivas, sem articulação à rede assistencial, não garantindo atenção integral. Nos outros casos, foi implementado como uma estratégia para mudar o modelo assistencial na atenção primária, substituindo total ou parcialmente as unidades básicas tradicionais preexistentes. Quando adotado como estratégia de mudança, foram observadas singularidades locais sugestivas das especificidades do processo de substituição da atenção básica pela equipe de saúde da família em grandes centros urbanos e da criatividade dos gestores locais; em alguns casos, o PSF desencadeou a reorga- nização do sistema de atenção, realizando-se como estratégia estruturante do sistema municipal.

Conclui-se que a implementação da estratégia de saúde da família em grandes centros urbanos difunde uma nova perspectiva para a atenção primária no Brasil, voltada para a família e a comunidade, que tem potencialidades para desencadear mudanças no modelo assistencial que efetivem o direito à saúde no cotidiano dos cidadãos. Os resultados positivos de algumas das experiências estudadas sugerem que o sucesso da implementação da estratégia para a grande maioria da população brasileira, afora incentivos federais, dependerá de estratégias criativas locais para enfrentar a diversidade de nosso País.

\section{SYNOPSIS}

\section{The Family Health Program and the construction of a new model for primary care in Brazil}

As part of the implementation of the country's Unified Health System (Sistema Único de Saúde), the Brazilian Government created, in the second half of the 1990s, the Family Health Program (FHP) (Programa de Saúde da Família), based on community-oriented, multidisciplinary care serving people organized into small groups. For this study, we evaluated the implementation of the FHP, based on three criteria: (1) the construction of the program as an entry point for most health needs and for access to specialized care, (2) the program's linkages with a comprehensive network of health services, and (3) the incorporation of new care practices into the health system. We found that the implementation of the FHP was far from uniform. In some municipalities the FHP is a focused program that runs in parallel with other primary care efforts. However, in other municipalities the FHP is viewed as a strategy aimed at changing the primary care model, and it partially or completely replaces preexisting primary care health units. Our research confirms a trend toward incremental change in the primary care model in Brazil. However, the expansion of the FHP in large urban areas faces several obstacles to guaranteeing all individuals access to comprehensive care with adequate clinical and collective health services, including secondary and tertiary care. The positive results that we found with some of the experiences with the FHP indicate that, in addition to increased federal financial incentives, the success of the FHP depends on creative local strategies to deal with Brazil's diversity.

Key words: primary health care, national health programs, delivery of health care, health services accessibility, Brazil. 


\section{REFERENCIAS}

1. Brasil, Ministério da Saúde. Manual para a organização da atenção básica. Brasília: Ministério da Saúde; 1998.

2. Paim JS. Descentralização das ações e serviços de saúde no Brasil e a renovação da proposta "Saúde para Todos". Rio de Janeiro: Universidade do Estado do Rio de Janeiro, Instituto de Medicina Social; 2002. (Série Estudos em Saúde Coletiva, 175).

3. Souza HM. Saúde da Família: desafios e conquistas. Em: Negri B, Viana AL, orgs. O Sistema Único de Saúde em dez anos de desafios. São Paulo: Sociedade Brasileira de Vigilância de Medicamentos/Centro de Estudos Augusto Leopoldo Ayrosa Galvão; 2002. Pp. 221-40.

4. Starfield B. Atenção primária: equilíbrio entre necessidades de saúde, serviços e tecnologia. Brasília: Unesco/Ministério da Saúde; 2002.

5. Brasil, Ministério da Saúde [site da Internet]. Disponível em: http://portal. saude.gov.br/saude/visao.cfm?id_area $=149$. Acessado em agosto de 2004 .

6. Viana AL, Dal Poz M. A reforma do sistema de saúde no Brasil e o Programa Saúde da Família. Physis (Rio J). 1998; 8(2):11-48.

7. Goulart F. Experiências em saúde da família: cada caso é um caso? [tese de doutorado]. Rio de Janeiro: Escola Nacional de Saúde Pública, Fundação Oswaldo Cruz; 2002

8. Sousa MF, Fernandes A, Araújo C, Fernandes MC. Gestão da atenção básica: redefinindo contextos e possibilidades. Divulgação em Saúde para Debate. 2000;21:7-14.

9. Escorel S, Giovanella L, Mendonça MHM, Magalhães R, Senna MCM. Avaliação da implementação do Programa Saúde da Família em dez grandes centros urbanos: síntese dos principais resultados. Brasília: Ministério da Saúde, Secretaria de Políticas de Saúde, Atenção Básica de Saúde; 2002.
10. Instituto Brasileiro de Geografia e Estatística. Censo demográfico 2000. Disponível em www.ibge.gov.br/cidadesat/ default.php. Acessado em junho de 2005.

11. Giovanella L, Escorel S, Mendonça MHM. Porta de entrada pela atenção básica? Integração do PSF à rede de serviços de saúde. Saude Debate. 2003; 27(65):278-89.

12. Canesqui AM, Oliveira AMF. Saúde da Família: modelos internacionais e estratégia brasileira. Em: Negri B, Viana $\mathrm{AL}$, orgs. O Sistema Único de Saúde em dez anos de desafios. São Paulo: Sociedade Brasileira de Vigilância de Medicamentos/Centro de Estudos Augusto Leopoldo Ayrosa Galvão; 2002. Pp. 241-69.

13. Brasil, Ministério da Saúde. Avaliação normativa do Programa Saúde da Família no Brasil. Monitoramento da implantação e funcionamento das equipes de saúde da família-2001-2002. Brasília: Ministério da Saúde; 2004.

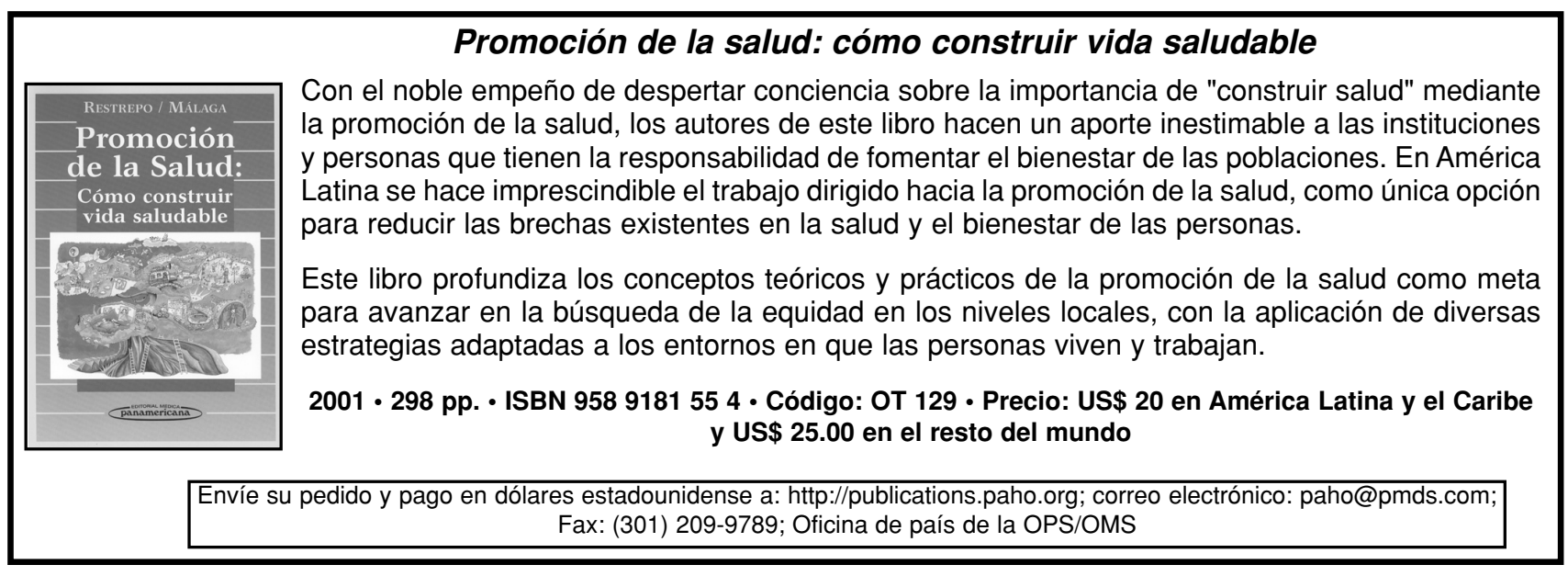

